\title{
Exploring the Potential of High Resolution Satellite Imagery for Yield Prediction of Avocado and Mango Crops ${ }^{\dagger}$
}

\author{
Moshiur Rahman 1,*, Andrew Robson ${ }^{1}$, Surantha Salgadoe ${ }^{1}$, Kerry Walsh ${ }^{2}$ and Mila Bristow ${ }^{3}$ \\ 1 Applied Agricultural Remote Sensing Centre (AARSC), University of New England, \\ Armidale, NSW 2351, Australia; andrew.robson@une.edu.au (A.R.); asalgado@myune.edu.au (S.S.) \\ 2 Institute of Future Farming Systems, Central Queensland University, Rockhampton, QLD 4701, Australia; \\ k.walsh@cqu.edu.au \\ 3 Department of Primary Industry and Resources, Northern Territory Government, \\ Darwin, NT 0828, Australia; Mila.Bristow@nt.gov.au \\ * Correspondence: mrahma37@une.edu.au \\ + Presented at the Third International Tropical Agriculture Conference (TROPAG 2019), Brisbane, Australia, \\ 11-13 November 2019.
}

Published: 7 April 2020

\begin{abstract}
Accurate pre-harvest yield estimation of high value fruit tree crops provides a range of benefits to industry and growers. Currently, yield estimation in Avocado (Persea americana) and Mango (Mangifera indica) orchards is undertaken by a visual count of a limited number of trees. However, this method is labour intensive and can be highly inaccurate if the sampled trees are not representative of the spatial variability occurring across the orchard. This study evaluated the accuracies of high resolution WorldView (WV) 2 and 3 satellite imagery and targeted field sampling for the pre-harvest prediction of yield. A stratified sampling technique was applied in each block to measure relevant yield parameters from eighteen sample trees representing high, medium and low vigour zones (6 from each) based on classified normalised difference vegetation index (NDVI) maps. For avocado crops, principal component analysis (PCA) and non-linear regression analysis were applied to 18 derived vegetation indices (VIs) to determine the index with the strongest relationship to the measured yield parameters. For mango, an integrated approach of geometric (tree crown area) and optical (spectral vegetation indices) data using artificial neural network (ANN) model produced more accurate predictions. The results demonstrate that accurate maps of yield variability and total orchard yield can be achieved from WV imagery and targeted sampling; whilst accurate maps of fruit size and the incidence of phytophthora can also be achieved in avocado. These outcomes offer improved forecasting than currently adopted practices and therefore offer great benefit to both the avocado and mango industries.
\end{abstract}

Keywords: yield prediction; WorldView-3 (WV3); avocado (Persea americana); mango (Mangifera indicea)

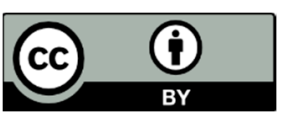

(C) 2020 by the authors. Licensee MDPI, Basel, Switzerland. This article is an open access article distributed under the terms and conditions of the Creative Commons Attribution (CC BY) license (http://creativecommons.org/licenses/by/4.0/). 